\title{
(6) OPEN ACCESS \\ Lumen-apposing metal stents (LAMS) for pancreatic fluid collection (PFC) drainage: may not be business as usual
}

\author{
Ji Young Bang, Muhammad Hasan, Udayakumar Navaneethan, Robert Hawes, \\ Shyam Varadarajulu
}

- Additional material is published online only. To view please visit the journal online (http://dx.doi.org/10.1136/ gutjnl-2016-312812).

Center for Interventional Endoscopy, Florida Hospital, Orlando, Florida, USA

\section{Correspondence to}

Dr Shyam Varadarajulu, Center for Interventional Endoscopy, Florida Hospital, 601 East Rollins Street, Orlando, FL 32803, USA;

svaradarajulu@yahoo.com

Received 6 August 2016 Revised 9 August 2016 Accepted 10 August 2016 Published Online First 31 August 2016

\section{MESSAGE}

Lumen-apposing metal stents (LAMS) have been recently developed to improve treatment outcomes in the endoscopic management of pancreatic fluid collections (PFC), particularly in walled-off necrosis (WON), to facilitate better drainage of necrotic contents and minimise the risk of perforation and peritoneal leakage. In an ongoing randomised trial, we observed serious adverse events that included delayed bleeding, buried stent syndrome and biliary stricture that necessitated a change in the management protocol for patients with PFC treated with LAMS.

\section{IN MORE DETAIL}

Randomised trials comparing endoscopic and surgical techniques for the management of PFCs have favoured the endoscopic approach. ${ }^{12}$ Endoscopy is less expensive, associated with shorter hospital stay and the clinical outcomes are comparable. To compare the clinical outcomes of patients undergoing endoscopic drainage of WON using LAMS or plastic stents, a randomised trial (NCT02685865) was initiated at our institution. Included in the study were patients with symptomatic WON measuring $>6 \mathrm{~cm}$ in size and located adjacent to the gastric or duodenal lumen. Excluded were patients with pseudocysts, WON inaccessible for endoscopic ultrasound-guided drainage and irreversible coagulopathy. The LAMS (Hot AXIOS, Boston Scientific) used in this trial had a single-step cautery-tipped delivery system with dimensions of $15 \mathrm{~mm}$ (diameter) by $10 \mathrm{~mm}$ (length). In the plastic stent cohort, two $7 \mathrm{Fr}$ by $4 \mathrm{~cm}$ double pigtail stents were deployed after dilation of the transmural tract to $12 \mathrm{~mm}$. Per study protocol, a follow-up CT was obtained at 4-6 weeks after the index intervention to assess treatment response with treatment success defined as resolution of WON to $<3 \mathrm{~cm}$ in conjunction with symptom relief. The main outcome measure was to compare the median number of interventions required to achieve treatment success. The secondary outcome measures were to compare the rates of treatment success and clinical and stent-related adverse events. The total sample size was estimated at 62 patients.

In this ongoing trial (started in February 2016), 21 patients have been randomised so far to LAMS CrossMark

To cite: Bang JY, Hasan M, Navaneethan U, et al. Gut 2017;66:2054-2056. $(n=12)$ or plastic stents $(n=9)$. A decision was made to conduct an interim audit because of a higher than anticipated procedural adverse event rate. Stent-related adverse events were observed in six of 12 patients randomised to LAMS compared with none in the plastic stent cohort $(50.0$ vs $0 \%$, $\mathrm{p}=0.019$ ). The adverse events included delayed bleeding after hospital discharge in three patients, buried stent syndrome in two and obstructive jaundice secondary to stent-induced biliary stricture in one. All adverse events were observed beginning 3 weeks after the index intervention. An emergent CT scan performed at the time of the adverse event occurrence revealed WON resolution in all six patients.

\section{Bleeding}

Three patients presented with severe GI bleeding requiring intensive care unit admissions and blood transfusions at $3 \quad(n=1)$ and 5 weeks $(n=2)$ post-LAMS placement. On endoscopic ultrasound (EUS) examination, interlacing vessels were visualised within the distal flange of the LAMS (figure 1, see online supplementary video 1 ), with CT angiograms confirming pseudoaneurysms in all three patients. All patients were successfully managed by interventional radiology (IR)-guided coil embolisation.

\section{Buried LAMS syndrome}

In two patients, the LAMS were found buried under the gastric mucosa on fluoroscopy when they presented for routine outpatient endoscopic stent removal at 5 and 6 weeks post-index procedure. In one of the patients, endoscopic stent retrieval resulted in massive haemorrhage requiring IR-guided coil embolisation. In the other patient, the buried LAMS was retrieved after transmural tract dilation using large diameter biopsy forceps (figure 2A, B, see online supplementary video 2 ).

\section{Biliary stricture}

One patient presented with obstructive jaundice at 5 weeks post-LAMS placement. At endoscopic retrograde cholangiopancreatography (ERCP), he had a distal biliary stricture secondary to mechanical compression induced by the LAMS that had been deployed via the duodenal bulb. The LAMS was removed and the patient was treated successfully at ERCP by the placement of a single $10 \mathrm{Fr}$ plastic biliary stent (figure $2 \mathrm{C}$, see online supplementary video 3 ).

\section{COMMENTS}

In this ongoing study, we observed a high rate of adverse events in patients with WON treated using LAMS. None of these adverse events were encountered intraprocedurally but rather manifested late or were evident only at the time of clinical follow-up. 


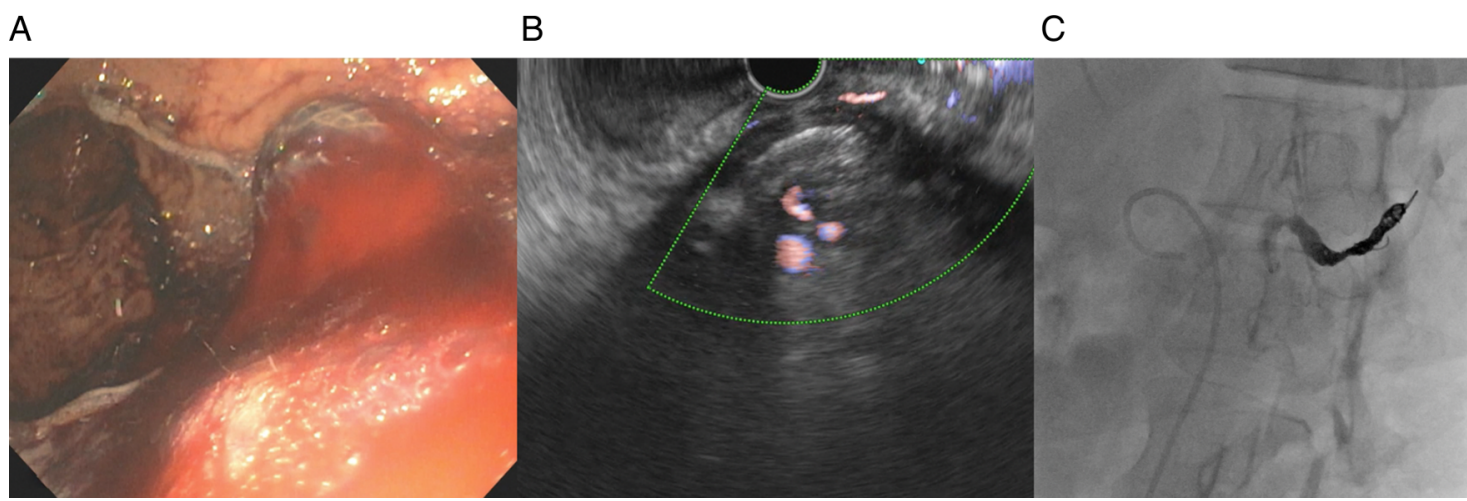

Figure 1 Endoscopic view revealed blood in the lumen-apposing metal stent $(A)$ with EUS revealing interlacing vessels at the distal flange (B), which was treated by interventional radiology-guided coil embolisation (C).

A

B

C

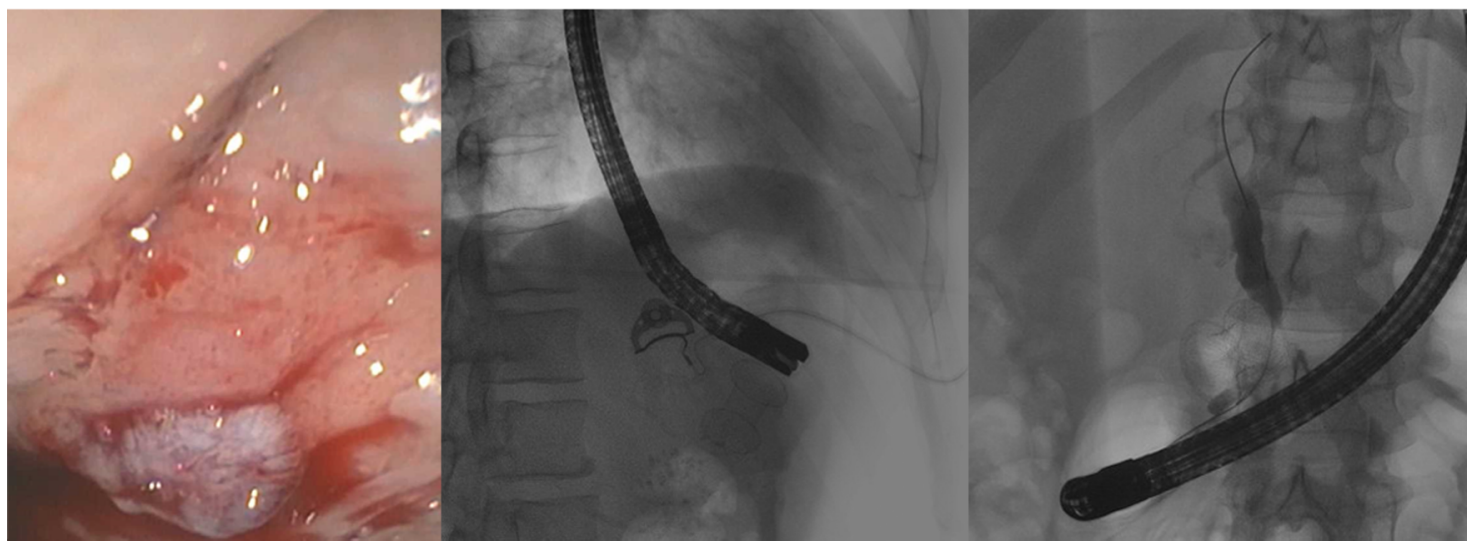

Figure 2 Endoscopic view revealed mucosal overgrowth (A) over a buried lumen-apposing metal stent (LAMS), which was visualised at fluoroscopy (B). Fluoroscopic image in a patient presenting with obstructive jaundice revealed a distal biliary stricture on cholangiogram and the LAMS compressing the distal bile duct (C).

Bleeding is a well-recognised intraprocedural adverse event encountered in patients undergoing endoscopic transmural drainage of PFCs. ${ }^{3}$ Late bleeding when observed is mostly due to underlying coagulopathy. In a recent meta-analysis that compared the metal and plastic stents for endoscopic transmural drainage of PFCs, there was no difference in the rate of haemorrhage between the two stent groups. ${ }^{4}$ However, the majority of patients in the metal stent cohort were treated using fully covered biliary metal stents and not LAMS. Furthermore, the number of patients in the fully covered metal stent cohort were small compared with the plastic stent cohort and as a result, the true incidence of adverse events related to the use of fully covered metal stents is not known. All three patients in our study presented with delayed bleeding at weeks $3(n=1)$ and 5 $(n=2)$ after placement of the LAMS. Based on our preliminary data, we hypothesise that plastic stents likely gravitate towards the GI lumen as the PFC resolves, whereas LAMS remain in place with the resultant friction against regional vasculature surrounding the necrotic cavity precipitating bleeding (figure 3). This was confirmed by EUS and CT angiography in all three patients.

In two patients who presented for follow-up endoscopy at weeks 5 and 6 after LAMS placement, we observed gastric mucosal overgrowth leading to buried stent syndrome. In a recent case series of two patients who had undergone LAMS placement for PFC and gallbladder drainage, buried stent syndrome was observed at 3 and 4 months, respectively. ${ }^{5}$ Unlike the long, freely mobile, double pigtail, plastic stents, LAMS are short $(10 \mathrm{~mm})$ and fairly immobile. Therefore, following PFC resolution, by virtue of their lumen-apposing property, the stent may become deeply embedded in the gastric wall layers with mucosal overgrowth resulting in buried stent syndrome. Removal of the buried stent was technically challenging in both of our patients. After passage of a guidewire via an ERCP cannula, the transmural tract was dilated to $12 \mathrm{~mm}$ using a radial expansion balloon. Attempts at endoscopic retrieval using rat-tooth forceps and a $15 \mathrm{~mm}$ stone extraction balloon were both unsuccessful and only the use of large $8 \mathrm{~mm}$ biopsy forceps was successful in retrieving the buried LAMS. However, this precipitated massive bleeding in one patient that required IR-guided coil embolisation.

In one patient who presented with obstructive jaundice at 5 weeks post-LAMS placement, the LAMS was found to be compressing the distal common bile duct. It is likely that after WON resolution, the distal flange of the LAMS impinged on the bile duct impeding bile flow. To our knowledge, this LAMS-related adverse event has not been previously reported.

Traditionally, patients with PFCs have been treated mainly with 7 or $10 \mathrm{Fr}$, double pigtail, plastic stents and have undergone a follow-up CT scan at 6 weeks to assess treatment response. In the present study, all adverse events were observed from 3 weeks after LAMS placement or were evident at 6-week 

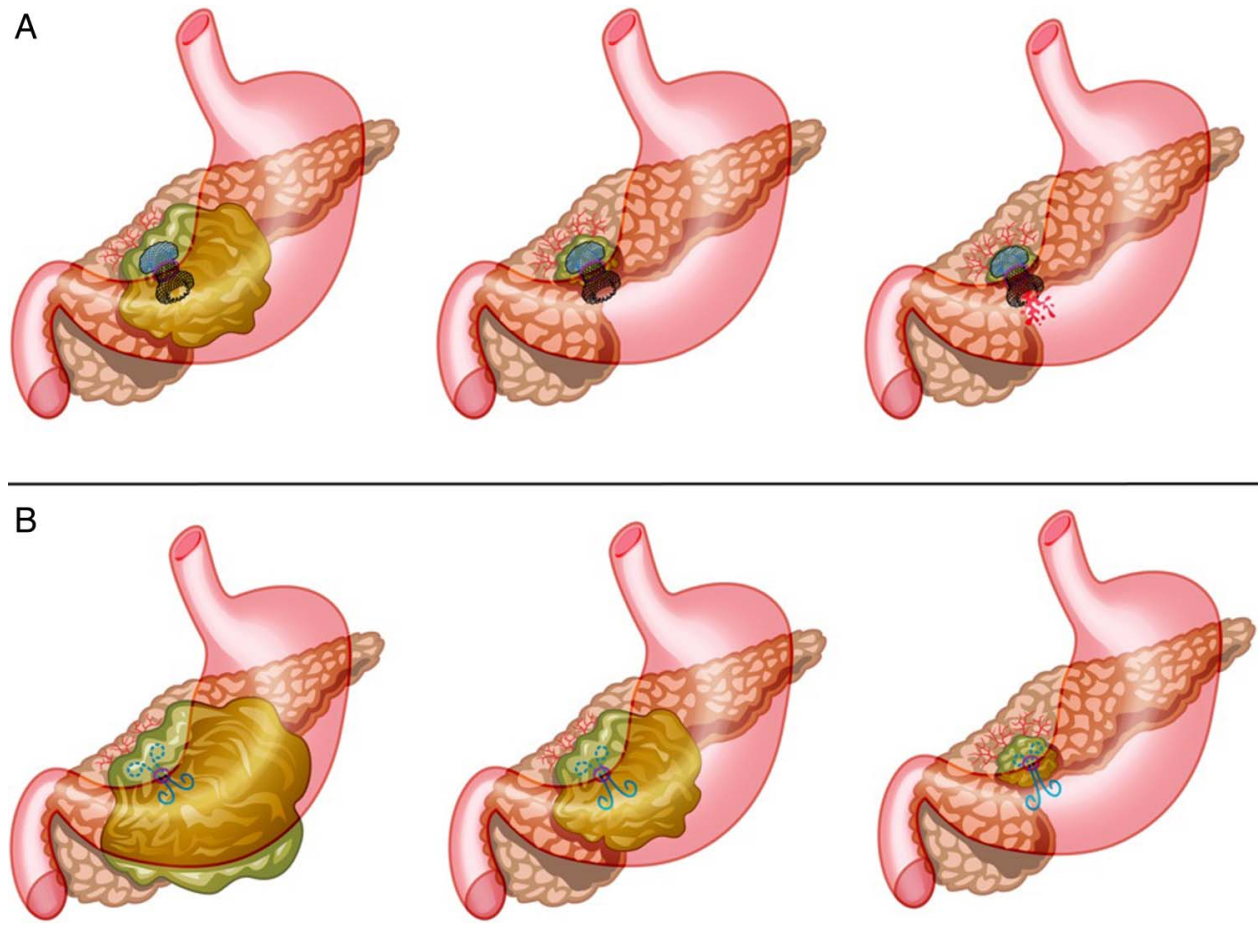

Figure 3 Illustration of the concept of delayed bleeding with the use of lumen-apposing metal stent (LAMS). The LAMS remains in situ with collapse of the walled-off necrosis cavity and precipitates bleeding (A), whereas the freely mobile double pigtail plastic stents gravitate towards the gastric lumen (B).

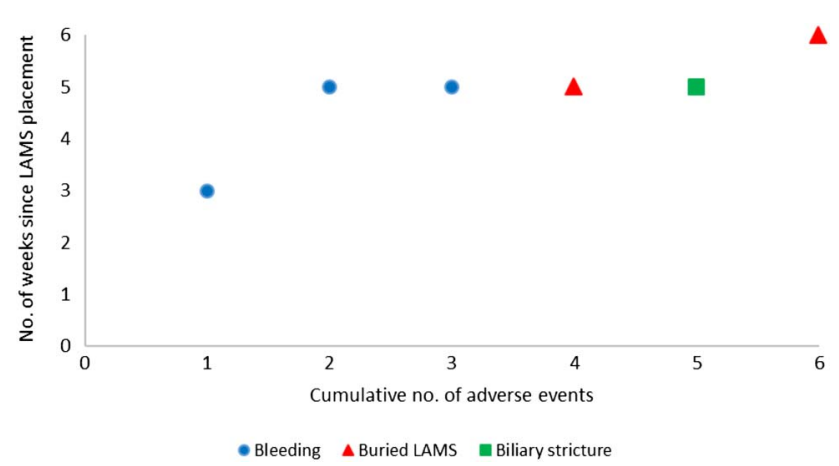

Figure 4 Scatter plot showing the incidence of adverse events following lumen-apposing metal stent (LAMS) insertion over time.

follow-up (figure 4). We hypothesise that the wide diameter of the LAMS facilitates better drainage of the necrotic contents leading to faster resolution of the PFC. However, once the PFC resolves, the LAMS by virtue of its immobility impinges on the adjacent vasculature or organs leading to bleeding, occlusion or becomes deeply buried in the gastric wall layers. Therefore, unlike in patients treated with plastic stents, to minimise adverse events and maximise treatment effects, a follow-up CT scan to assess treatment response must be obtained at three, rather than 6 weeks, followed by LAMS removal if the PFC has resolved. Alternatively, given the high costs associated with CT imaging, transabdominal ultrasound could be an alternative imaging modality to assess treatment response.

These preliminary but important observations prompted a change in our study and clinical practice protocols whereby a CT scan is now obtained at 3 weeks in all patients treated with LAMS followed by stent removal if the PFC has resolved. We believe that these observations are important given the increasing use of LAMS for PFC drainage in recent times.

Contributors JYB: analysis and interpretation of the data, drafting of the article, critical revision of the article for important intellectual content and final approval of the article. $\mathrm{MH}, \mathrm{UN}$ and $\mathrm{RH}$ : endoscopist performing the procedure, critical revision of the article for important intellectual content and final approval of the article. SV: conception and design, interpretation of data, drafting of the article, critical revision of the article for important intellectual content and final approval of the article.

Competing interests SV and RH are consultants for Boston Scientific Corporation and Olympus America Inc.

Ethics approval Institutional review board.

Provenance and peer review Not commissioned; internally peer reviewed.

Open Access This is an Open Access article distributed in accordance with the Creative Commons Attribution Non Commercial (CC BY-NC 4.0) license, which permits others to distribute, remix, adapt, build upon this work non-commercially, and license their derivative works on different terms, provided the original work is properly cited and the use is non-commercial. See: http://creativecommons.org/ licenses/by-nc/4.0/

\section{REFERENCES}

1 Varadarajulu S, Bang JY, Sutton BS, et al. Equal efficacy of endoscopic and surgical cystogastrostomy for pancreatic pseudocyst drainage in a randomized trial. Gastroenterology 2013;145:583-90.

2 Bakker OJ, van Santvoort HC, van Brunschot S, et al. Endoscopic transgastric vs surgical necrosectomy for infected necrotizing pancreatitis: a randomized trial. JAMA 2012;307:1053-61.

3 Vazquez-Sequeiros E, Baron TH, Pérez-Miranda M, et al, Spanish Group for FCSEMS in Pancreas Collections. Evaluation of the short- and long-term effectiveness and safety of fully covered self-expandable metal stents for drainage of pancreatic fluid collections: results of a Spanish nationwide registry. Gastrointest Endosc 2016;84:450-7.

4 Bang JY, Hawes R, Bartolucci A, et al. Efficacy of metal and plastic stents for transmural drainage of pancreatic fluid collections: a systematic review. Dig Endosc 2015;27:486-98.

5 Fabbri C, Luigiano C, Marsico $\mathrm{M}$, et al. A rare adverse event resulting from the use of a lumen-apposing metal stent for drainage of a pancreatic fluid collection: "the buried stent". Gastrointest Endosc 2015;82:585-7. 